\title{
Physiologische und ethische Implikationen in Schopenhauers Konzept der ästhetischen Kontemplation
}

\author{
Brigitte Scheer \\ Johann Wolfgang Goethe-Universität - Frankfurt am Main (Deutschland)
}

\begin{abstract}
ZUSAMMENFASSUNG
Ein Hauptziel dieses Essays ist es, aufzuzeigen, dass Schopenhauers reine ästhetische Kontemplation zwar eine außergewöhnliche, aber keine übernatürliche Erkenntnisweise darstellt. Um dies zu beweisen, bedarf es der Offenlegung einer gewissen Affinität von Körper (Physis) und Geist, wie sie Schopenhauer für das Verständnis der reinen Kontemplation in seiner Theorie der menschlichen Sinne (insbesondere Gesichtssinn und Gehör) darlegt. Die aktive und objektive Ausrichtung dieser Sinne ist bereits eine Bedingung für die ethisch bedeutsame gelegentliche Überwindung des Willens in der ästhetischen Kontemplation.
\end{abstract}

\section{ABSTRACT}

One of the main purposes of this essay is to show that Schopenhauer's concept of a pure aesthetic contemplation indeed is an extraordinary mode of cognition but not a supernatural one. To demonstrate this, means to reveal that there is in Schopenhauer's concept of pure aesthetic contemplation a certain affinity of body (physis) and mind (Geist) which he develops in his theory of the human senses (especially of sight and hearing). The active and objective orientation of these senses is already a condition of the ethical impact of the pure aesthetic contemplation in its occasional suppression of the will.

Im Titel meines Vortrags wird eine Verbindung von physiologischen und ethischen Komponenten in Schopenhauers Konzept der ästhetischen Kontemplation unterstellt. Das mutet zunächst provokativ an, weil diese Verbindung womöglich sogar widersprüchlich ist: Denn der Leib, die Physis, ist Schopenhauer zufolge die Objektivation des Willens, also eine Manifestation von Lebensäußerungen, die nur dem Selbst zuarbeiten. Ethisches Verhalten kann dagegen allein in der Überwindung des Egoismus entstehen. Nun ist bekannt, dass Schopenhauer zufolge die ästhetische Kontemplation zu einem Zustand der Selbstvergessenheit führt. Sind damit die physiologischen Voraussetzungen der ästhetischen Kontemplation schon als irrelevant anzusehen?

Meines Erachtens sind die physiologischen Prozesse ästhetischer Wahrnehmung für den schliesslichen Verlauf der reinen ästhetischen Kontemplation von großer Wichtigkeit, und zwar nicht nur in ihrer gewöhnlichen allgemeinen Form der Sinneswahrnehmungen, sondern in der spezifischen Weise, die Schopenhauer nicht allen Menschen, sondern nur den genialisch Veranlagten 
zuspricht. Dies gilt vor allem für den ästhetisch Produktiven, das heißt für den Künstler, der seine Eingebungen für das Kunstwerk aus der intensivierten Anschauung der Dinge, das heißt aus ästhetischer Kontemplation gewinnt. Es gilt aber auch für den kongenialen Betrachter von Kunst und Natur, der seine reine interessenfreie Anschauung so weit steigern kann, dass er über der Schau des Wesens der Dinge sein Selbst vergessen kann. In diesen Fällen lässt sich sagen, dass geradezu mit den Mitteln der menschlichen Physis das Leibbewusstsein aufgehoben wird. Dies ist nur denkbar aufgrund einer Affinität von Physis und Geist.

Die ästhetische Kontemplation ist ein Schauen der Ideen, die Schopenhauer als die ewigen Formen versteht, die in den Objektivationsstufen des Willens, das heißt in den Gattungen der natürlichen Dinge und Kräfte realisiert sind. Die Formen selber, als unmittelbare Objektität des Willens unterliegen nicht dem Prinzip aller Endlichkeit, das heißt dem Satz vom Grund. Sie verlangen zu ihrer Erkenntnis demnach auch nicht, wie in gewöhnlicher empirischer Erkenntnis, ein Subjekt als Individuum, dessen Vorstellungen durch einen Leib und seine Affektionen vermittelt sind. Die Idee erfordert vielmehr $\mathrm{zu}$ ihrer Vergegenwärtigung das Korrelat des reinen Subjekts der Erkenntnis.

Im zweiten Buch seines Hauptwerks hatte Schopenhauer das Konzept eines für sich bestehenden rein erkennenden Subjekts als ,geflügelter Engelskopf ohne Leib" verspottet. (W I, 118) Muss er nun das Subjekt ästhetischer Kontemplation dieser Verspottung aussetzen? Ich möchte in meinem Beitrag zeigen, dass Schopenhauer die reine ästhetische Kontemplation zwar als außergewöhnliche, aber nicht als übernatürliche Erkenntnisweise herleitet.

Schopenhauer folgt Kant in der Forderung, das ästhetische Wohlgefallen müsse frei von Interessen und Zweckgebundenheit sein. Anders als Kant, der ein begriffsloses Wohlgefallen propagiert und damit die ästhetische Erfahrung subjektiv bleiben lässt, räumt Schopenhauer dem Subjekt ästhetischer Kontemplation die Möglichkeit ein, zur unmittelbaren Objektität des Willens, das heißt zur Erkenntnis der Ideen vorzudringen. Dies soll in reiner Anschauung geschehen, das heißt, ohne dass das Angeschaute einen unmittelbaren Anreiz für den Willen böte.

Aus meiner Sicht kommt es darauf an, schon in Schopenhauers allgemeiner Theorie der Anschauung genuine Vorbedingungen aufzuzeigen, die jene reine 
Anschauung oder Kontemplation erklärlich machen, in der das Individuum sich zum reinen Subjekt wandelt und seinen Willen, das heißt seinen Leib, nicht mehr empfindet. $\mathrm{Zu}$ den Vorbedingungen gehört die von Schopenhauer immer wieder betonte Intellektualität schon der gewöhnlichen Anschauung durch die Operation des Verstandes. Hier beweist sich eine Anschlussfähigkeit des Intellekts an die Sinnlichkeit, wie sie in der ästhetischen Betrachtung ebenfalls notwendig wird. Zu deren Voraussetzung gehört im einzelnen die spezifische Verfasstheit der menschlichen Sinnesorgane, zu deren Erforschung Schopenhauer umfangreiche physiologische Beiträge liefert. Schopenhauers eigene Untersuchungen bestätigen am Ende die Bewertungen der Sinne, wie sie traditionell vorgenommen wurden, wobei ihm jedoch neue Einsichten aus der Sinnesphysiologie zu Gebote stehen. Hiernach gibt es Sinne mit besonderer Affinität zum Willen, das heißt zum Leib, nämlich Geschmack, Geruchssinn und Tastsinn, und solche, die dem Willen ferner stehen und gelegentlich als „theoretische Sinne“ bezeichnet werden, nämlich Gehör und Gesichtssinn.

Für das Verständnis einer möglichen rein ästhetischen, das heißt selbstzweckhaften Betrachtung der Welt kommt es darauf an, diese außergewöhnliche Wahrnehmung als im Intellekt und in den Sinnesorganen angelegt zu erweisen. Genau hierum bemüht sich Schopenhauer mit seiner Theorie der menschlichen Sinne.

Die Dignität von Gesichtssinn und Gehör begründet Schopenhauer zunächst willenstheoretisch dadurch, dass beide Sinne nicht unmittelbar zur Befriedigung von Leibbedürfnissen fungieren. In späteren Schriften, das heißt im Ergänzungsband zum Hauptwerk, in Schopenhauers Farbenlehre und in der Schrift «Über den Willen in der Natur» zeigt Schopenhauer seine Genugtuung darüber, dass die neueren physiologischen Erkenntnisse über die Sinnesfunktionen die eigenen theoretisch gewonnenen Einsichten voll bestätigen. Der Gesichtssinn ist Schopenhauer zufolge der am meisten objektive. Er liefert dem Verstand das meiste Material zum Aufbau der Welt der Vorstellungen. Darin erweist er sich als der aktive Sinn. Die Sinnesphysiologie bestätigt das objektive, von sich selbst abständige Wesen des Gesichtssinnes. Es lässt sich nämlich nachweisen, dass beim Sehvorgang keine unmittelbaren Empfindungen von Annehmlichkeit oder Unannehmlichkeit im Sehorgan selbst entstehen. Es gibt beim primären Sehvorgang keine unmittelbare Organempfindung wie beim Hören. So zeigt sich 
ansatzweise eine Leibvergessenheit beim Sehvorgang als solchem und macht die Extremform der Leibüberwindung in der ästhetischen Kontemplation verständlicher. Den aktiven Charakter des Sehvorgangs verdeutlicht Schopenhauer im Vergleich mit dem passiven Gehörssinn. Er erläutert diesen Unterschied damit, „daß das Hören vermöge einer mechanischen Erschütterung des Gehörnerven vor sich geht, die sich sogleich bis ins Gehirn fortpflanzt; während hingegen das Sehn eine wirkliche Aktion der Retina ist, welche durch das Licht und seine Modifikationen bloß erregt und hervorgerufen wird.“(W II, 33)

Die Sonderstellung des Gesichtssinns äußert sich Schopenhauer zufolge neben seiner Aktionsfähigkeit auch darin, dass er ein Fernsinn und ein Sinn äußerster Sensibilität ist. Schopenhauer erklärt: „Das Gesicht hat den ersten Rang, sofern seine Sphäre die am weitesten reichende, und seine Empfänglichkeit die feinste; was darauf beruht, daß sein Anregendes ein Imponderabile, d.h. ein kaum noch Körperliches, ein quasi Geistiges, ist.“ (W II, 32) Gemeint ist das Licht, das Schopenhauer „das Erfreulichste der Dinge“ nennt. (W I, 235) Die Freude und Beglückung, die das Licht und mehr noch die transparenten, lichtdurchfluteten Farben gewähren, sind darauf zurückzuführen, „daß das Licht das Korrelat und die Bedingung der vollkommensten anschaulichen Erkenntnißweise ist, der einzigen, die unmittelbar durchaus nicht den Willen afficirt." (W I, 235) Es geht um die intuitive Erkenntnis der Platonischen Ideen, die das Subjekt nur dann erreichen kann, wenn es sich vom Dienst am Willen befreit, das heißt die Bedürfnisse des Leibes nicht mehr empfindet und seine Individualität vergisst. Wenn man sich vergegenwärtigt, dass der menschliche Leib „durch und durch Wille ist“, wie Schopenhauer betont, so ist es - wiederum mit seinem Wort - „,bewundernswerth“ (W II, 31), dass eine interessenfreie reine Anschauung in ästhetischer Kontemplation überhaupt möglich ist. Den Schlüssel zu dieser Möglichkeit findet Schopenhauer unter anderem in der Physiologie des Gesichtssinnes, dessen Sehnerv gegenüber Leibempfindungen gleichgültig bleibt. Damit ist eine relative Emanzipation des Gesichtssinnes von leiblicher Bestimmung erfolgt und zumindest der Anfang einer rein objektiven Betrachtung gewonnen. Es ist interessant $\mathrm{zu}$ sehen, dass Schopenhauer im 1. Band seines Hauptwerks diese physiologische Besonderheit nur dem Gesichtssinn zugesprochen hat, während er im späteren Ergänzungsband auch dem Gehörssinn diese relative Abständigkeit vom Leib eingeräumt hat. Wenn sich die gehörten Töne in einem akustischen 
Normalbereich bewegen, so lautet die Begründung, ist auch das Hören frei von unmittelbaren Empfindungen der Annehmlichkeit oder Unannehmlichkeit, also in leibneutraler Wahrnehmung verfahrend.

Dass es sinnliche Wahrnehmungen gibt, in die sich das Wollen oder die Bedürfnisse des Leibes nicht unmittelbar hineindrängen, ist nicht nur von ästhetischem, sondern von deutlichem ethischen Belang: Die momentane Aufkündigung der Dienstbarkeit am Willen wird zugleich als Möglichkeit der Askese erfahren und als zeitweise Aufhebung des Leidens, das aus dem chronischen Wollen erwächst. Die Verneinung des Willens als ethisches Verhalten erfährt den Vorschein ihrer eigenen Möglichkeit am Beispiel der ästhetischen Kontemplation.

Die verstärkte Sensibilität der Sinne ist eine notwendige, aber noch nicht hinreichende Bedingung für das Ereignis der ästhetischen Kontemplation. Schopenhauer zufolge bedarf es hierfür weiterer physiologischer und ideeller Voraussetzungen, die sich nur bei den „höher Begabten“ (P II, 3) das heißt bei den genialen Menschen finden. Sie zeichnen sich generell durch eine Erkenntnisabsicht aus, die auf das rein Objektive abzielt. Diese Veranlagung zählt Schopenhauer zum Wesen des Genies, während der gewöhnliche Mensch den Willensbezug, das heißt den Rückbezug auf das empirische Subjekt in allem Erkennen nicht überwindet. So bleibt es bei den gewöhnlichen Menschen bei der Erkenntnis bloßer Relationen der Dinge untereinander und zum Subjekt; über das Erfassen des Individuellen reicht diese Erkenntnis nicht hinaus. Das Genie dagegen begnügt sich nicht mit dem Erkennen relationaler Verhältnisse, sondern forscht dem Wesen der Dinge selbst nach, und dies nicht mit den Mitteln der Begrifflichkeit, sondern auf dem Weg der intensivierten Anschauung, die ja aus Schopenhauers Sicht die genuine Quelle aller Erkenntnis ist. Üblicherweise verlassen die Menschen die Anschauungstätigkeit, sobald sie einen Ansatz zur Nutzung und Verarbeitung des Angeschauten bemerken. Das Genie dagegen verharrt zu Zeiten bei der Anschauung mit solchen Kräften des Intellekts, dass sich ihm über die unmittelbare Erscheinung hinaus das Wesen des angeschauten Dinges offenbart. Schopenhauer macht für diese Tätigkeit nicht nur bewusste Absichten verantwortlich, sondern ebenso gehirnphysiologische Besonderheiten beim genialisch Begabten. Er erklärt: „was aber diesen Zustand [gemeint ist die reine ästhetische Anschauung. v. Verf.] ausnahmsweise herbeiführt, müssen innere 
physiologische Vorgänge seyn, welche die Thätigkeit des Gehirns reinigen und erhöhen, in dem grade, daß eine solche plötzliche Springfluth derselben entsteht.“ (W II, 426) Über die Veränderung im Subjekt der reinen Anschauung bemerkt Schopenhauer an anderer Stelle, sie entspringe ,physiologisch betrachtet, aus einer starken Erregung der anschauenden Gehirnthätigkeit, ohne alle Erregung der Neigungen oder Affekte.“(W II, 419)

Das Gehirn konzentriert also offensichtlich seine ganze Kraft auf die Anschauung, so dass es keine anderen Empfindungen über die Bild- oder Gestaltwahrnehmung hinaus mehr zulässt. Es kommt hierbei zur Schau einer Form, die der empirischen Erscheinung als Muster zugrunde liegt. Diese Form ist jedoch nicht abstrakt, sondern gestalthaft vorzustellen. Sie verkörpert den Typus einer bestimmten Gattung von Erscheinungen. Schopenhauer nennt sie Idee im „Wohlverstandenen“ Sinn der Platonischen Idee. Das subjektive Korrelat dieser Ideenschau verändert sich plötzlich im Vollzug seines Schauens, indem es seine Leibempfindung einbüßt und sich nicht mehr als Individuum weiß, das heißt, es vergisst seinen Willen und ist von seinem Gegenüber so erfüllt, dass es mit ihm Eins wird. Das bedeutet, dass sein Bewusstsein allein das Geschaute zu seinem Inhalt hat. Dessen unmittelbare Sinnlichkeit wird transparent für das anschaubare Allgemeine der Idee. Mit Schopenhauers Worten ist der Schauende hierbei „reines, willenloses, schmerzloses, zeitloses Subjekt der Erkenntnis“ geworden. (W I, 211)

Die so beschriebene Einswerdung von Subjekt und Gegenstand als höchste Form der Erkenntnis hat ihr Vorbild bei den Mystikern der Philosophiegeschichte, aber Schopenhauer kommt es darauf an. Diese Einswerdung mit den eigenen systematischen Mitteln zu erläutern. So erklärt er: „Als Wille, außer der Vorstellung und allen ihren Formen, ist er einer und der selbe im kontemplirten Objekt und im Individuo, welches sich an dieser Kontemplation emporschwingend als reines Subjekt seiner bewußt wird.“ (W I, 212)

Die ästhetische Kontemplation ermöglicht Schopenhauer zufolge die höchste und eigentliche Erkenntnis, nämlich die Antwort auf die Frage nach dem Was oder nach dem Wesen der Dinge. Die Antwort wird gewissermaßen zweispurig erteilt; auf dem Weg der Willensmetaphysik und auf dem Weg der physiologischen Wissenschaft. Schopenhauer nutzt die Einsichten der materialistischen Physiologie seiner Zeit als ein wesentliches Mittel zur 
Realisierung seines Ideals einer sich auf die Empirie stützenden Metaphysik. Über das Verhältnis von Physiologie und Metaphysik äußert Schopenhauer in seinem Werk «Über den Willen in der Natur» folgendes Resümee:

\begin{abstract}
Die wahre Physiologie, auf ihrer Höhe, weist das Geistige im Menschen (die Erkenntniß) als Produkt seines Physischen nach: [...] aber die wahre Metaphysik belehrt uns, daß dieses Physische selbst bloßes Produkt, oder vielmehr Erscheinung, eines Geistigen (des Willens) sei. (N, Ausg. Die kleinen Schriften, Löhneysen, 340)
\end{abstract}

Die Prädikate, die dem Subjekt der ästhetischen Kontemplation beigefügt werden, weisen darauf hin, dass dessen Erfahrung nicht nur im epistemischen oder ästhetischen Kontext von Bedeutung ist. Der Zustand der zeitweisen Willenlosigkeit und Schmerzlosigkeit ist am Ende für Schopenhauer deutlich wichtiger als die Erkenntnisfreude und das Wohlgefallen am Schönen, welches ja ebenfalls legitime Momente der reinen ästhetischen Betrachtung sind. Die ethische Bedeutung einer zumindest zeitweisen Möglichkeit der Ausschaltung des Wollens und damit des Leidens an der Endlichkeit und Bedürftigkeit des Menschen ist klar herausgestellt. Es geht hier um die Vorzeichnung einer Erlösung vom Leiden, die nur durch die Aufhebung des Wollens denkbar ist, wie es unter anderem der Asket und der Heilige in Schopenhauers Ethik erkennen lassen. Wie sehr Schopenhauer seine Metaphysik des Schönen selbst als Beitrag zur Ethik versteht, lässt eine Äußerung aus seiner Vorlesung über die Metaphysik des Schönen erkennen. Es heißt dort: „Der Zustand des reinen völlig willenlosen Erkennens ist es [...] ganz allein, der uns ein Beispiel giebt, von der Möglichkeit eines Daseyns, das nicht im Wollen besteht, wie unser jetziges.“ (Met. D. S., 96) 